\title{
A luta contra o envelhecimento, uma análise na perspectiva bioética
}

\author{
The struggle against aging, a bioethical analysis \\ La lucha contra el envejecimiento, un análisis bioético
}

Recebido: 21/01/2021 | Revisado: 24/01/2021 | Aceito: 24/01/2021 | Publicado: 31/01/2021

Olivia Figueira

ORCID: https://orcid.org/0000-0002-1531-3072 Pontifícia Universidade Católica do Paraná, Brasil E-mail: olivia2407@yahoo.com.br

Jociane Casellas

ORCID: https://orcid.org/0000-0003-2352-6526 Pontifícia Universidade Católica do Paraná, Brasil E-mail:jocicasellas1975@gmail.com

Alan Figueira

ORCID: https://orcid.org/0000-0002-1557-6059 Hospital Naval Marcilio Dias, Brasil E-mail: alan_figueira@hotmail.com

Carla Corradi Perini

ORCID: https://orcid.org/0000-0002-9340-8704 Pontifícia Universidade Católica do Paraná, Brasil E-mail: carla.corradi@pucpr.br

\begin{abstract}
Resumo
Vivemos em uma sociedade que se recusa a envelhecer. As exigências estéticas impostas pela sociedade, de aparência sempre jovial, negando ou escondendo os sinais que o passar do tempo impõe atingem diretamente as pessoas idosas, de forma estigmatizante, e tornam suas possibilidades diminuídas pelo sentimento de não pertencimento. Objetivo: O objetivo deste artigo é explorar as estratégias declaradas na luta contra o envelhecimento. Busca-se analisar os aspectos que apoiam as estratégias e discutir o fenômeno do combate ao envelhecimento na perspectiva bioética. Metodologia: Trata-se de uma pesquisa qualitativa que utilizou como material de análise o conteúdo de domínio público disponível na internet, em sua maioria visando o ideal estético. A pesquisa realizou coleta direta de dados com o pesquisador como o principal instrumento, e realizou análise de conteúdo de Bardin. Conclusão: Atualmente as estratégias declaradas na luta contra o envelhecimento, em sua maioria visam o ideal estético, como forma de permitir a pessoa idosa a permanência no meio social. São estratégias para disfarçar o envelhecimento ou tentar impedi-lo em uma busca incessante da juventude eterna.
\end{abstract}

Palavras-chave: Envelhecimento; Bioética; Envelhecimento saudável; Vulnerabilidade.

\begin{abstract}
We live in a society that refuses to grow old. The aesthetic demands imposed by society, to be always youthful in appearance, denying or hiding the signs that the passage of time imposes, directly affect elderly people in a stigmatizing way, having their possibilities diminished by the feeling of not belonging. Objective: The objective of this article is to explore the strategies declared in the struggle against aging. It seeks to analyze the aspects that support the strategies and discuss the phenomenon of combating aging from the perspective of bioethics. Methodology: This is a qualitative research that used the public domain content available on the internet as material for analysis, which mostly aims at the aesthetic ideal. The research performed direct data collection with the researcher as the main instrument and performed Bardin's content analysis. Conclusion: Currently the strategies declared in the struggle against aging aim an aesthetic ideal, to allow the elderly to remain in the social environment. They are strategies to disguise aging or try to prevent it in an incessant search for eternal youth.
\end{abstract}

Keywords: Aging; Bioethics; Healthy aging; Vulnerability.

\section{Resumen}

Vivimos en una sociedad que se niega a envejecer. Las exigencias estéticas impuestas por la sociedad, que siempre son de apariencia juvenil, negando u ocultando los signos que impone el paso del tiempo, afectan directamente a las personas mayores de manera estigmatizante, que tiene sus posibilidades disminuidas por el sentimiento de no pertenencia. Objetivo: El objetivo de este artículo es explorar las estrategias declaradas en la lucha contra el envejecimiento. Se busca analizar los aspectos que sustentan las estrategias y discutir el fenómeno de la lucha contra el envejecimiento desde la perspectiva de la bioética. Metodología: Se trata de una investigación cualitativa que utilizó el contenido de dominio público disponible en Internet como material de análisis, que apunta principalmente al ideal estético. La investigación realizó la recolección directa de datos con el investigador como instrumento principal y realizó el análisis de contenido de Bardin. Conclusión: Actualmente las estrategias declaradas en la lucha contra el 
envejecimiento, en su mayoría apuntan al ideal estético, como una forma de permitir que la persona mayor permanezca en el entorno social. Son estrategias para disfrazar el envejecimiento o intentar prevenirlo en una búsqueda incesante de la eterna juventud.

Palabras clave: Envejecimiento; Bioética; Envejecimiento saludable; Vulnerabilidad.

\section{Introdução}

Vivemos em uma sociedade que se recusa a envelhecer, valorizando o vigor físico e a beleza associada à juventude, que não aceita o processo de envelhecimento, vinculando-o ao sofrimento, solidão, decrepitude e morte. Entretanto, a velhice inclui uma dimensão existencial, pois transforma a relação do indivíduo com o tempo e afeta sua relação com o mundo. O ser humano, ao rejeitar a morte, acaba por rejeitar também a velhice, talvez por essa fase da vida ser a que mais se aproxima da morte, a velhice se torne uma ameaça para a vida (Lira Harris \& Guimarães Protti, 2016).

Envelhecer é processo natural para aqueles que tem a oportunidade de atingir essa fase do ciclo vital. Homens e mulheres parecem negar o envelhecimento, que é parte desse ciclo: nascer, crescer, envelhecer e morrer. Cada indivíduo possui uma imagem corporal de si mesmo, e essa visão se transforma em cada estágio da vida podendo-se encontrar no corpo de um sujeito as expressões de si e de sua trajetória (Santos et al., 2020). Atualmente, a juventude e sua força são os componentes essenciais no contexto de uma sociedade que faz do corpo o instrumento de expressão e construção de identidade (Marinho \& Kairós, 2016) e ao se analisar a relação da estética com o envelhecimento, tem-se a perda do forte, saudável, produtivo e valoroso, para a entrada do frágil, impotente, improdutivo e dependente, caracterizações essas produzidas socialmente.

O envelhecimento é um processo condicionado por fatores biológicos, sociais, econômicos, culturais, ambientais e históricos, podendo ser definido como um processo progressivo de mudança biopsicossocial da pessoa durante todo o ciclo de vida. Um dos desafios ao se avaliar o envelhecimento é que muitas percepções e suposições comuns sobre pessoas mais velhas são baseadas em estereótipos ultrapassados (Organização Mundial da Saude, 2015). As exigências estéticas impostas pela sociedade, de aparência sempre jovial, negando ou escondendo os sinais naturais que o passar do tempo impõe, atingem diretamente as pessoas idosas.

$\mathrm{Na}$ atualidade, mais e mais pessoas idosas frequentam salões de beleza, clínicas de cirurgias plásticas, centros de estética entre outros, levando ao questionamento do porquê: será por vaidade ou por medo de aceitar o envelhecimento? Será devido à cobrança social e pessoal de não deixar transparecer o avançar da idade? Por que a repulsa ao envelhecimento? Por que expressões como antienvelhecimento, anti-idade e luta contra o envelhecimento se tornaram tão comuns e populares nos dias de hoje? Através de uma pergunta simples podemos perceber o quão arraigado está à recusa ao envelhecimento na nossa sociedade: Quais as estratégias declaradas na luta contra o envelhecimento?

Assim, o objetivo deste artigo é explorar as estratégias declaradas na luta contra o envelhecimento. Especificamente, busca-se analisar os aspectos basilares que apoiam as estratégias e discutir o fenômeno do combate ao envelhecimento na perspectiva da bioética.

\section{Metodologia}

Trata-se de uma pesquisa qualitativa, que tem por característica a importância da interpretação por parte do pesquisador com suas opiniões sobre o fenômeno em estudo, neste caso, o envelhecimento(Soares et al., 2018). A pesquisa realizou com coleta direta de dados com o pesquisador como o principal instrumento, e realizou análise de conteúdo de Bardin (Mendes \& Miskulin, 2017). Utilizou-se como material o conteúdo de domínio público disponível na internet, tendo como questão norteadora: Quais as estratégias declaradas nessa "luta contra o envelhecimento?". O material foi submetido à análise de conteúdo de Bardin que consiste em 3 etapas: 1-pré análise; 2-exploração do material; 3-tratamento dos resultados e 
interpretação. Na primeira etapa, pré-análise, é feita a organização do material com leitura flutuante, escolha dos documentos a serem analisados, formação de hipóteses e objetivos. Na segunda etapa, de exploração do material, é feita a codificação e seleção das unidades de registro, que agrupadas formam os núcleos de sentido para a posterior categorização. Na terceira etapa é feito o tratamento dos resultados e interpretação por meio de inferência.

Inicialmente realizou-se uma busca livre no Google em 13/07/2020, com a expressão "luta contra envelhecimento", sendo recuperadas 3.510.000 citações. Foram selecionadas as 20 primeiras citações em ordem de aparecimento, mencionadas nos resultados por um código alfanumérico (pi1, pi2...). As 20 páginas foram acessadas, sendo o conteúdo lido integralmente (pré-análise). Na etapa de exploração do material, a leitura foi novamente realizada, buscando responder à questão norteadora, de onde emergiram as 26 unidades de registros, agrupadas em 6 núcleos de sentido, que compuseram as 2 categorias apresentadas neste estudo.

\section{Resultados}

As categorias que emergiram da análise de conteúdo são: "A luta contra o envelhecimento baseada na estética", que englobou 3 núcleos de sentido e 12 unidades de registo apresentadas no Quadro 1, e "Não lutar contra, mas aceitar o envelhecimento" composta por 3 núcleos de sentido e 14 unidades de registo, apresentada no Quadro 2. Cada núcleo de sentido teve o seguinte quantitativo de referências: A-7 B-5 C-3 D-3 E-1 F-1.

Quadro 1. Núcleos de sentido e unidades de registros que compõe a Categoria "A luta contra o envelhecimento baseada na estética".

\begin{tabular}{|c|c|}
\hline $\begin{array}{ll}\begin{array}{l}\text { Núcleo } \\
\text { Registro }\end{array} & \text { de } \\
\end{array}$ & Unidades de Registro \\
\hline $\begin{array}{l}\text { A - Oferta de } \\
\text { tratamento } \\
\text { medicamentoso } \\
\text { / estético contra } \\
\text { o } \\
\text { envelhecimento }\end{array}$ & $\begin{array}{l}\text { "verdadeira fórmula da juventude" pi4 } \\
\text { "o martírio de aparentar velhice" pi1 } \\
\text { "lidar com a dura realidade de envelhecer" pi4 } \\
\text { "reverter sintomas de envelhecimento" pi4 } \\
\text { "melhorar aspecto da pele reduzindo sinais da idade" pi8 } \\
\text { "remover a aparência de envelhecimento e recuperar a vitalidade e luminosidade da juventude" } \\
\text { pi15 } \\
\text { "terapia anti-idade e antienvelhecimento" pi18 }\end{array}$ \\
\hline $\begin{array}{l}\text { B - Métodos } \\
\text { não invasivos } \\
\text { para evitar o } \\
\text { envelhecimento }\end{array}$ & $\begin{array}{l}\text { "ioga contra o envelhecimento do rosto" pi13, pil6 } \\
\text { "rotina alimentar antienvelhecimento para uma pele firme e jovial" pi14 } \\
\text { "recuperação da vitalidade" pi14 } \\
\text { "alimentos antioxidantes para adiar o aparecimento das marcas de expressão" pi18 }\end{array}$ \\
\hline $\begin{array}{l}\text { E - Estudo } \\
\text { científico sobre } \\
\text { envelhecimento } \\
\text { facial e sua } \\
\text { associação com } \\
\text { tabagismo }\end{array}$ & $\begin{array}{l}\text { "O tabagismo causa envelhecimento facial, e isso seria uma argumentação e motivação efetiva } \\
\text { para parar de fumar" pi10 }\end{array}$ \\
\hline
\end{tabular}

Fonte: Figueira (2020).

Neste quadro observamos como palavras de cunho pejorativas são utilizadas para estimular a luta contra o envelhecimento. Através de colocações como "martírio", “dura realidade”, e similares, os idosos são forçados, ou pelo menos convidados, a reconhecer sua decrepitude, o que os mantem subjugados a um ideal que não compõe sua realidade. Tal característica demonstra o peso do preconceito social contra o envelhecimento. 
Quadro 2. Núcleos de sentido e unidades de registros que compõe a Categoria "Não lutar contra, mas aceitar o envelhecimento".

\begin{tabular}{|c|c|}
\hline Núcleo de Registro & Unidades de Registro \\
\hline $\begin{array}{l}\text { C - Análise } \\
\text { sociocultural do } \\
\text { envelhecimento }\end{array}$ & $\begin{array}{l}\text { "temos um ideal estético atual sem rugas, sem nenhum sinal de envelhecimento" pi2 } \\
\text { "na cultura atual o binômio que define a beleza é juventude" pi2 } \\
\text { "o problema é que envelhecemos, mas não podemos mostrar nossa velhice" pi9 } \\
\text { "nossa maior contradição é querermos viver mais, porém envelhecer é intolerável" pi9 } \\
\text { "só envelhece quem quer, ou seja, só fica velho quem não investe pesadamente para aparentar } \\
\text { jovialidade" pi9 } \\
\text { "velhofobia" pi19 } \\
\text { "esses preconceitos, abusos psicológicos, estigma que os velhos sofrem desde sempre" pi19 } \\
\text { "a quantidade de pessoas que realmente acredita que os velhos são descartáveis, inúteis e } \\
\text { improdutivos, e que podem morrer é assustadora" pi19 }\end{array}$ \\
\hline $\begin{array}{l}\text { D - Suporte para } \\
\text { lidar com o } \\
\text { envelhecimento }\end{array}$ & $\begin{array}{l}\text { "não é possível frear o envelhecimento e impedir as mudanças que ocorrem com a idade, mas é } \\
\text { possível aumentar a eficiência da parte que sobrevive" pi7 } \\
\text { "devemos estar preparados para aceitar e entender o enfraquecimento do corpo e buscar o } \\
\text { fortalecimento da alma" pi6 } \\
\text { "tentar minimizar o poder da insatisfação contra o envelhecimento faz parte do crescimento mental" } \\
\text { pi6 } \\
\text { "dicas e conteúdos sobre o envelhecimento e a velhice. Afinal, seremos idosos uma única vez" pi11 }\end{array}$ \\
\hline $\begin{array}{l}\text { F - Plano de ação } \\
\text { internacional para } \\
\text { envelhecimento }\end{array}$ & $\begin{array}{l}\text { "o combate à discriminação, à negligência, ao abuso e aos maus tratos à velhice" pi20 } \\
\text { "o desafio de viver com dignidade, uma vida socialmente ativa durante todo o percurso do } \\
\text { envelhecimento, é uma realidade que envolve a todos nós" pi20 }\end{array}$ \\
\hline
\end{tabular}

Fonte: Figueira (2020).

Neste quadro podemos observar críticas sendo feitas ao ideal social predeterminado para o idoso, e propostas para que as pessoas idosas revejam sua posição diante da sociedade. Aceitar o envelhecimento faz parte da aceitação de algo que é imutável, e inevitável, a passagem do tempo. São feitas sugestões de como e por que aceitar esse processo que é natural para o ser humano.

\section{Discussão}

O envelhecimento passou por uma série de mudanças conceituais ao longo da história mundial chegando à visão atual. No século XIX surgiram e foram se definindo separações em grupos etários, relacionando-se a funções sociais, hábitos e espaços ocupados na sociedade, e por isso supõe-se que o conceito de envelhecimento se relaciona de forma muito próxima ao curso de modernização e revolução industrial.

No século XX se segue o processo de modificação sociocultural do envelhecimento, muito alinhado com a definição médica de pessoa idosa estipulada pela geriatria e gerontologia que se iniciavam, e traziam as definições que foram usadas como base também para a criação de políticas públicas específicas. A velhice até então vista como uma etapa de preparação e espera da morte teve sua visão modificada, com os discursos sobre envelhecimento ativo e capacidade funcional, já não mais associada a doença, mas a habilidade de executar atividades do cotidiano, começando a separação da velhice como acontecimento natural, fisiológico, e da doença, processo patológico (Helena \& Bernardo, 2019).

A Organização Mundial de Saúde define envelhecimento saudável como o processo de desenvolvimento e manutenção da capacidade funcional que permite o bem-estar em idade avançada, ele não é definido por um nível ou limiar específico do funcionamento, e sim pela experiência individual, na sua subjetividade (Organização Mundial da Saude, 2015). Envelhecer bem e ter uma velhice com qualidade é um processo que depende de diversos fatores que interagem entre si. Neste sentido, o envelhecimento é uma das expressões de um ser que está em movimento, integrado com o mundo que o envolve, que o constitui e que é produzido por ele (Furtado Nogueira \& Bloc Boris, 2019). A velhice potencializa limitações no que diz 
respeito a saúde, as relações sociais, ao manejo da vida diária e do status social, e por isso, muito é exigido do ponto de vista adaptativo, e a não-adaptação pode gerar um desequilíbrio entre emoções, interesses, bem-estar, imagem social e envolvimento global com a vida. Envelhecer com qualidade não depende somente de bem-estar físico, e da redução de doenças, mas também de fatores psicossociais que implicam na ação de toda a sociedade e de instituições públicas (Piñas, 2020). Atualmente, observa-se um flagrante problema entre o corpo e as tecnologias, conflitando os conceitos de saúde e beleza, na medida em que inexiste um marco para que se possa determinar o que é saúde e o que vem a ser beleza (Carvalho, 2018). Os sinais do envelhecimento constrangem as pessoas idosas, pois se distanciam do padrão considerado ideal, e isso faz com que tendam a resistir a estas modificações, investindo em produtos e serviços para modificar a aparência corporal (Cordeiro et al., 2017).

É importante lembrar dos artigos 8 e 10, respectivamente, do Estatuto do Idoso (Saúde, 2003); que o envelhecimento é um direito personalíssimo e a sua proteção um direito social. Que fala também sobre a garantia da liberdade, respeito e dignidade, como pessoa humana e sujeito de direitos civis, políticos, individuais e sociais. Refere o direito ao respeito que consiste na inviolabilidade da integridade física, psíquica e moral, abrangendo a preservação da imagem, da identidade, da autonomia, de valores, ideias e crenças. A dignidade da pessoa idosa é assinalada como instrumento promotor de cidadania, saúde, cuidados e qualidade de vida, para que a mesma tenha condições de alcançar a longevidade e uma existência digna (da Luz Cabral, 2017).

Além das vulnerabilidades genético-biológicas, estamos expostos e somos suscetíveis ao longo da vida aos resultados do efeito que as influências socioculturais tem em cada um (Sathler et al., 2007). A inatividade no envelhecimento pode tornar patológicos os aspectos fisiológicos do envelhecer (Figueira et al., 2019), entretanto, envelhecer não significa uma perda completa dos papéis sociais, e sim uma modificação dos mesmos. O isolamento da pessoa idosa é um reflexo da falta de identificação das pessoas com o outro e a fragilidade dessas é muitas vezes suficiente para separar os que envelhecem, ou seja, sua decadência as isola (Cecília \& Minayo, 2017). As condições que limitam o acesso a experiências que fazem a pessoa idosa sentir-se bem e descrever-se como altamente satisfeitos e envolvidos com a vida podem ser deletérias (Sathler et al., 2007).

Atualmente a bioética pode ser entendida como sendo uma reflexão complexa, interdisciplinar e compartilhada sobre a adequação das ações envolvendo a vida e o viver (Andrade et al., 2016). É complexa, porque inclui os múltiplos aspectos envolvidos no seu objeto de atenção, interdisciplinar devido à possibilidade de contar com conhecimentos advindos das mais variadas áreas do saber. Compartilhada, por utilizar as diferentes interfaces para realizar diálogos mutuamente enriquecedores (Goldim, 2009). Ela é a convergência de diversos saberes, de forma multi, trans e interdisciplinar.

A Declaração Universal sobre Bioética e Direitos Humanos (DUBDH) (UNESCO, 2006), assinada por 191 países em 2005, reconheceu os direitos humanos como referencial mínimo e universal para a bioética. Ela parte do reconhecimento de que a saúde é resultante de múltiplos aspectos que englobam não só o progresso científico e tecnológico, mas também fatores culturais e sociais (Rojas Cruz et al., 2010). Em seu artigo 11, a DUBDH propõe: "Nenhum indivíduo ou grupo deve ser discriminado ou estigmatizado por qualquer razão, o que constitui violação à dignidade humana, aos direitos humanos e às liberdades fundamentais". O estigma só se produz ou se concretiza quando retirado do outro a sua dignidade, diminuído naquilo que o constitui como ser humano (Godoi \& Garrafa, 2014). Os padrões de beleza são fatores sociais criados para padronizar e ditar as importâncias socioculturais criando estigmas e marcas irreparáveis (Fort et al., 2017).

Todo ser humano possui dignidade como valor absoluto, sem discriminação de raça, cultura, religião, estado de saúde, condições socioeconômicas ou etárias (Luiz et al., 2010). Para Kant, aquilo que é um fim em si mesmo não tem meramente valor relativo ou preço, mas um valor interno, isto é, dignidade. Kant afirma que "o homem não tem preço", em outras palavras, a dignidade humana se justifica pelo fato dela não ter preço. A partir dessa ótica, a afirmação de Kant constitui um referencial teórico para que se possa estabelecer um processo de conscientização em torno da dignidade e dos direitos da 
pessoa idosa, e serve como ponto de partida e referencial teórico para o combate a ações que violam tais direitos (Pereira, 2018).

Para Beauchamp e Childress, no princípio da beneficência algumas regras são necessárias, como proteger e defender os direitos dos outros, evitar que sofram danos, eliminar condições que causarão danos, e socorrer pessoas que estão em perigo. O princípio da beneficência aplicado à população idosa envolve aceitar o envelhecimento como uma etapa positiva, com suas vantagens e desvantagens, assim como todas as outras etapas da vida, e passar a defender o direito da pessoa idosa de "ser idoso e idosa" em sua integralidade, que não precise mais disfarçar a idade, e que possa abraçá-la. Aceitar as modificações físicas que ocorrem com o envelhecimento é uma forma de respeito e de preservação da dignidade da pessoa que envelhece, uma vez que a estigmatização desumaniza. O corpo considerado velho é aquele que não se encontra de acordo com os parâmetros determinados socialmente, e, portanto, são ofertados práticas e procedimentos que objetivam torná-lo culturalmente adequado, a fim de atender as exigências consideradas ideais. Os padrões estéticos definidos por uma conjuntura social acabam por dimensionar um obstáculo ao reconhecimento do indivíduo e a sua real identidade (Fin et al., 2017).

A dignidade constitui um valor imutável e intangível que não pode depender das circunstâncias existenciais concretas, nem ser subordinada ao juízo de ninguém. A defesa da dignidade humana é um princípio central na bioética, e por isso é importante eliminar a discriminação e estigmatização da pessoa idosa, o que contribui para o aumento da sua vulnerabilidade.

\section{Conclusão}

Ao aceitar o processo de envelhecimento deixa-se de vulnerar, de violar a dignidade da pessoa idosa, esta que é um ser humano, com toda sua complexidade, um ser bio-psico-socio-espiritual com múltiplas dimensões que constituem sua integralidade.

A pessoa estigmatizada tem suas possibilidades diminuídas pelo sentimento de não pertencimento, aumentando sua vulnerabilidade, e prejudicando sua autoestima. A estigmatização fere a autonomia da pessoa idosa como indivíduo e cabe a nós, como sociedade, a não estigmatização. Atualmente as estratégias declaradas na luta contra o envelhecimento, em sua maioria visam o ideal estético, como forma de permitir a pessoa idosa a permanência no meio social. São estratégias para disfarçar o envelhecimento ou tentar impedi-lo em uma busca incessante da juventude eterna. Trabalhos futuros que tragam propostas e meios de combater a estigmatização inclusive pelo olhar da pessoa idosa são necessários.

\section{Referências}

Andrade, A. F. L. D., Pessalacia, J. D. R., Daniel, J. C., \& Euflauzino, I. (2016). Processo ensino-aprendizagem em bioética: um debate interdisciplinar. SciELO Brasil. https://www.scielo.br/scielo.php?pid=S0100-55022016000100102\&script=sci_arttext\&tlng=pt

Carvalho, A. (2018). Hábitos de beleza e saúde:"Paradigmas da beleza." Revista.Universo.Edu.Br. http://www.revista.universo.edu.br/index.ph $\mathrm{p}$ ?journal=1UNIVERSOSALVADOR2\&page=article\&op=view\&path\%5B $\% 5 \mathrm{D}=5703$

Cecília, M., \& Minayo, S. (2017). Violência contra idosos $O$ avesso do respeito à experiência e à sabedoria. http://www.observatorionacionaldoidoso.fiocruz.br/biblioteca/_manual/4.pdf

Cordeiro, R., Pereira, S., A. B.-R. A., \& 2017, U. (2017). Velha é a vovozinha: uma análise interpretativa do consumo e estigma associado à identidade de idade de mulheres após os 65 anos. Periodicos.Estacio.Br. http://periodicos.estacio.br/index.php/admmade/article/view/2957

da Luz Cabral, M. (2017). Longevidade: Cidadania, Participação e Direitos Sociais. Ri.Ucsal.Br, 1, 18-31. https://doi.org/10.17058/psiunisc.v1i1.9629

Figueira, O., Figueira, H., Figueira, J., Health, A. G.-, \& 2019, U. (2019). Depression in Climacteric Women: Most Meaningful Symptoms. Scirp.Org. https://www.scirp.org/journal/paperinformation.aspx? paperid=92149

Fin, T., Portella, M., \& Scortegagna, S. (2017). Velhice e beleza corporal das idosas: conversa entre mulheres. SciELO Brasil. https://www.scielo.br/scielo.php?pid=S1809-98232017000100074\&script=sci_arttext\&tlng=pt

Fort, M. C., Skura, I., \& Brisolara, C. (2017). Convenções corporais e o medo de envelhecer: Ideais de juventude e beleza midiáticos versus aceitação pessoal e social da imagem real. Revista Observatório, 3(1), 183-204. https://sistemas.uft.edu.br/periodicos/index.php/observatorio/article/view/3071 
Research, Society and Development, v. 10, n. 1, e56210112254, 2021 (CC BY 4.0) | ISSN 2525-3409 | DOI: http://dx.doi.org/10.33448/rsd-v10i1.12254

Furtado Nogueira, C., \& Bloc Boris, D. (2019). Envelhecimento na perspectiva fenomenológico-existencial de Sartre e de Beauvoir. Revistadematematicas.Uchile.Cl, 28(2), 1-14. https://doi.org/10.5354/0719-0581.2019.55661

Godoi, A. M. M., \& Garrafa, V. (2014). Leitura bioética do princípio de não discriminação e não estigmatização. Saúde e Sociedadede e Sociedade, 23(1), 157-166. https://www.scielosp.org/article/sausoc/2014.v23n1/157-166/

Goldim, J. (2009). Bioética complexa: uma abordagem abrangente para o processo de tomada de decisão. AMRIGS.

Helena, M., \& Bernardo, J. (2019). Expressões Conservadoras no Campo Geriatrico/Gerontologico: O Enfoque Comportamental em Debate. In Anais Congresso Brasileiro de Assistentes Sociais 2019. 16(1).

Lira Harris, P., \& Guimarães Protti, G. (2016). Velhice e envelhecimento: experiências de idosos em unidades de terapia inten siva. In Arq Med Hosp Fac Cienc Med (Vol. 61). http://189.125.155.35/index.php/AMSCSP/article/view/126

Luiz, D., Ramos, P., \& Lucato, M. C. (2010). O Conceito de Pessoa Humana da Bioética Personalista. In bioetica.org.br. http://www.bioetica.org.br/library/modulos/varias_bioeticas/arquivos/varias_personalista.pdf

Marinho, M. dos S., \& Kairós, L. dos R. (2016). Velhice e aparência: a percepção da identidade de idosas longevas. Kairos Gerontologia, 19(1). https://revistas.pucsp.br/kairos/article/view/29479

Mendes, R. M., \& Miskulin, R. G. S. (2017). A análise de conteúdo como uma metodologia. Cadernos de Pesquisa, 47(165), 1044-1066. https://doi.org/10.1590/198053143988

Organização Mundial da Saude. (2015). Relatorio Mundial de Envelhecimento e Saúde. In Relatorio Mundial de Envelhecimento e Saúde.

Pereira, J. A. (2018). Uma discussão sobre a dignidade da pessoa humana a partir da ética de Kant. Griot: Revista de Filosofia, 17(1), 1-11. https://doi.org/10.31977/grirfi.v17i1.813

Piñas, A. (2020). Antropología del envejecimiento: el respeto a la autonomía del mayor. Mejorar el cuidado mediante la bioética narrativa Revistas.Comillas.Edu, 12,01-15. https://doi.org/10.14422/rib.i12.y2020.004

Rojas Cruz, M., Torres Oliveira, S. de L., \& Cordón Portillo, J. A. (2010). A Declaração Universal sobre Bioética e Direitos Humanos - contribuições ao Estado brasileiro. Revista Bioética. http://www.revistabioetica.cfm.org.br/index.php/revista_bioetica/article/view/538

Santos, I., von Humboldt, S., \& Leal, I. (2020). O efeito da imagem corporal e da satisfação conjugal no ajustamento ao envelhecimento dos idosos. Psicologia, Saúde \& Doença, 21(01), 111-116. https://doi.org/10.15309/20psd210117

Sathler, S., Batistoni, T., \& Liberalesso Neri, A. (2007). Percepção de classe social entre idosos e suas relações com aspectos emocionais do envelhecimento. Psicologia Em Pesquisa, 1(2), 3-10. http://periodicos.ufjf.br/index.php/psicologiaempesquisa/article/view/23687

Saúde, M. (2003). Estatuto do Idoso $3^{a}$ edição $2^{a}$ reimpressão. www.saude.gov.br/editora

Soares, A., Dorlivete, P., Shitsuka, M., Parreira, F. J., \& Shitsuka, R. (2018). Metodologia da Pesquisa Cientifica.

UNESCO. (2006). Declaração Universal sobre Bioética e Direitos Humanos - Biblioteca Digital da UNESCO. https://unesdoc.unesco.org/ark:/48223/pf0000146180_por 